\title{
Bi-dimensional Empirical Mode Decomposition Algorithm Based on Particle Swarm-Fractal I nterpolation
}

\author{
Feng-Ping $\mathrm{An}^{1,2}$, Xin $\mathrm{He}^{3}$ \\ ${ }^{1}$ School of Physics and Electronic Electrical Engineering, Huaiyin Normal University \\ Huaian, JS 223300-China \\ [e-mail: anfengping@163.com] \\ ${ }^{2}$ School of Information and Electronics, Beijing Institute of Technology \\ Beijing, BJ 100081-China \\ ${ }^{3}$ Institute of Intelligent Network System, Henan University, Kaifeng 475004, China \\ [e-mail: 271007963@qq.com] \\ *Corresponding author: Feng-Ping An, Xin He
}

Received March 7, 2018; revised May 18, 2018; accepted June 16, 2018;

published December 31, 2018

\begin{abstract}
Performance of the interpolation algorithm used in the technique of bi-dimensional empirical mode decomposition directly affects its popularization and application, so that the researchers pay more attention to the algorithm reasonable, accurate and fast. However, it has been a lack of an adaptive interpolation algorithm that is relatively satisfactory for the bi-dimensional empirical mode decomposition (BEMD) and is derived from the image characteristics. In view of this, this paper proposes an image interpolation algorithm based on the particle swarm and fractal. Its procedure includes: to analyze the given image by using the fractal brown function, to pick up the feature quantity from the image, and then to operate the adaptive image interpolation in terms of the obtained feature quantity. All parameters involved in the interpolation process are determined by using the particle swarm optimization algorithm. The presented interpolation algorithm can solve those problems of low efficiency and poor precision in the interpolation operation of bi-dimensional empirical mode decomposition and can also result in accurate and reliable bi-dimensional intrinsic modal functions with higher speed in the decomposition of the image. It lays the foundation for the further popularization and application of the bi-dimensional empirical mode decomposition algorithm.
\end{abstract}

Keywords: Fractal, Particle Swarm Algorithm, Bi-dimensional Empirical Mode Decomposition, Optimization, Image Interpolation 


\section{Introduction}

$\mathbf{D}_{\text {iscrete data interpolation is an important part of the application of bi-dimensional empirical }}$ mode decomposition (BEMD) to an image processing [1, 2]. At present, the bi-dimensional empirical mode decomposition algorithm requires discrete data interpolation estimation. In the bi-dimensional empirical mode decomposition algorithm, it is called surface interpolation. The purpose of the bi-dimensional empirical mode decomposition is to obtain a bi-dimensional intrinsic modal function (BIMF) that is more valuable. Under normal circumstances, this decomposition can ensure that the screening process is more optimized; The decomposition is mainly used to find the upper and lower envelopes after extracting the extreme points. Therefore, the precise envelope is highly important, and the interpolation calculation is focused on by many scholars' research and attention. Authors have proposed a number of interpolation methods, such as radial basis interpolation, cubic spline interpolation, and cubic interpolation [3-6]. Although these interpolation methods have an effect, they still have problems; and they cannot be based on image feature information for adaptive interpolation [9-17].

The surface interpolation needs to complete the interpolation operation in the bi-dimensional space, which causes the interpolation problem to become complicated. Furthemore, this interpolation is the basis and premise of image processing in the bi-dimensional empirical mode decomposition algorithm. The surface interpolation is also an important guarantee for the bi-dimensional inherent modal function of image decomposition. Therefore, for the bi-dimensional empirical mode decomposition of the interpolation algorithm, many scholars put forward the corresponding solutions, such as the following: In 2005, at the first university in Paris, Nunes proposed a radial basis method, that can be the interpolation fit, but it has low interpolation efficiency and low precision [1]; In 2007, at the Chinese Academy of Sciences Institute of Automation Liu Zhongxuan proposed the cubic interpolation method, which improved the interpolation accuracy, but still had low interpolation efficiency [18]; In 2011, at the Chongqing University Mathematics and Statistics College of Deng Lei proposed B-spline interpolation method, although the interpolation accuracy has been improved, but did not solve the problem of interpolation efficiency [19]; In 2015, the University of Regensburg, in Germany Saad et al [20] proposed a fast interpolation algorithm based on the Green function, but the interpolation effect was not ideal; In 2016, at the China University of Geosciences Institute of Automation Xu proposed the Kriging envelope interpolation method, which attempted to solve the interpolation problem and was applied to geochemical identification. But the computational efficiency was still low [21].

In addition, we can see that the existing interpolation methods can be interpolated in the bi-dimensional empirical mode for image processing, but they still have the following problems: First, the calculation efficiency is low; Second, the interpolation accuracy is low; Third, Image feature for adaptive interpolation. To this end, this paper first uses the fractal theory to adaptively extract the feature parameters of the image. Next, for the image interpolation, the interpolation parameters are processed through the particle group for optimization. Based on the solution of these problems, an interpolation algorithm based on fractal-particle swarm optimization is proposed, which can solve the problem of the computational efficiency and interpolation precision of the interpolation algorithm, and realize the optimal interpolation of the bi-dimensional empirical mode decomposition. 


\section{Fundamentals of Fractal Interpolation}

\subsection{Basic overview of fractal theory}

Fractal theory's developmental time has been relatively short. This theory is an important part of complex scientific theory and has affected people's ways of thinking and research methods. Fractal theory has presented new results regarding the informal, complex and irregular problems that traditional tools cannot describe. It also makes people have a new understanding of nature and many other fields, which also fully embodies the fractal theory of the simulation of structural capacity [22].

\subsection{Iterative functions and collage theorem}

The fractal interpolation algorithm has the two basic properties of the Iterated Function System (IFS) and the Collage Theorem. Among them, the iterated function system can guarantee the constancy of the attractor and use it to control the change of the image. The collage theorem can make the fractal dimension of an image based on fractal interpolation unchanged[23,24].

Definition 1: Let $X$ be a subset of the geometric space $R, d$ be a function of $X \times X$ to $R$, where the function $d: X \times X \rightarrow R$ satisfies the following condition:

1) Uniqueness: When $x=y, d(x, y)=0, x, y \in R$;

2) Symmetry: $d(x, y)=d(y, x), x, y \in R$;

3) Triangle inequality: $d(x, y)+d(y, z) \geq d(x, z), x, y, z \in R$;

Then, $d$ is a measure of $X$. $(x, y)$ represents a metric space with a metric $d$.

Definition 2: Assuming that $\left\{x_{n}\right\}$ is the column of the geometric space $X$, then it has a natural number $N$ for any $\varepsilon>0$, and when $m, n>N,\left|d\left(x_{m}, x_{n}\right)\right|<\varepsilon$, then the column $\left\{x_{n}\right\}$ is a Cauchy sequence.

The Cauchy sequence has the following two important properties [23, 24]:

(1) For the Cauchy sequence existing in a metric space, its limit is not necessarily in the same metric space;

(2) Any convergence sequence must be a Cauchy sequence, and any Cauchy sequence must be a bounded sequence.

Theorem 1: If $\left\{x_{n}\right\}_{n=1}^{\infty}$ is a sequence of metric space $(X, d)$, it converges to a point $x, x \in R$, then $\left\{x_{n}\right\}_{n=1}^{\infty}$ is a Cauchy sequence.

Definition 3: If any Cauchy sequence $\left\{x_{n}\right\}_{n=1}^{\infty}$ in the metric space $(X, d)$ has a limit value $x, X$ $\in R$, then this metric space $(X, d)$ has completeness.

If $(X, d)$ is a complete metric space, then any element $x$ in $X$ is changed to $x^{\prime}\left(x^{\prime} \in R\right)$ at function $d: X \times X \rightarrow R$. Any subspace of the complete space is complete if and only if it is a closed subset. The complete metric space indicates that the elements in the space converge to the same point. The difference between them is the convergence mode and convergence speed.

Here we call the intersection of all closed sets of set $\mathrm{A}$ as a closure of $\mathrm{A}$, denoted as $\bar{A}$; for all the union of the open sets contained in $A$, we call the interior of $A$, denoted as $\operatorname{int}(A)$. In other words, the closure of $\mathrm{A}$ is the minimum closed set of $A$, and the interior of $A$ is the largest 
open set contained in $A$, and the boundary of $A$ is denoted by $\partial A$ as defined below: $\partial A=\bar{A}-\operatorname{int}(A)$.

Definition 4: If the set $A, B(B \subset A \subset \bar{B})$ in the measurement space $(X, d)$ has a point in set $A$, it can be infinitely close to it in set $B$, then set $B$ is the thick subset of set $A$.

Definition 5: If the set $A$ in the $(X, d)$ metric space is compact, it is assumed that there is a finite number of open sets of open sets of any covering $A$.

Set $A$ is a tight indication that there is always another point in any small range around any point in set $A$.

Definition 6: Assuming that the metric space $(X, d)$ is completes the set $A, B$ is a subset of $X$. Thus the distance $d$ from set $A$ to set $B$ is defined as follows:

$$
d(A, B)=\max \{d(x, B) ; x \in A\}
$$

Where $d(x, B)=\min \{d(x, y) ; y \in B\}$.

From definition 6 we can see that this distance formula does not satisfy the exchange law requirement, which is $d(x, B)=\min \{d(x, y) ; y \in B\}$

Therefore, the distance defined by definition 6 cannot be used as a measure between set $A$ and set $B$.

Definition 7: Assuming that the metric space $(X, d)$ is complete, the set $A, B$ is a subset of the metric space $(X, d)$, and the Hausdorff distance between sets $A$ and $B$ is denoted as $h_{d}(A, B)$. The specific formula is as follows:

$$
h_{d}(A, B)=\max \{d(A, B), d(B, A)\}
$$

It can be seen from formula (2) that this distance formula satisfies the exchange law requirement.

The iteration function refers to the repeated function with their own complex, and the compound process is called an iteration. The iterations on the set $X$ are in the form of the following. Let $X$ be a set. The function $f$ is a mapping of $X \rightarrow X$, the $n$ iterations $f^{n}$ of the definition function $f$ are denoted as $f^{n}=f o f^{n-1}$, and o represents the compound $(f \circ g)(x)=f(g(x))$ of the function.

Definition 8: If $w_{n}: X \rightarrow X, n=1,2, \ldots, N$ is a finite squeezed family defined on the $(X, d)$ metric space. $n=1,2, \ldots, N$ will form a set of iterative functions, which are denoted as $X: w_{n}$, $n=1,2, \ldots, N$. If the compression ratio of $w_{n}$ is $c_{n}, n=1,2, \ldots, N$, then $c=\max \left\{c_{n}, n=1,2, \ldots, N\right\}$ is the compression ratio of this iterative function.

Theorem 2: Suppose that $(X, d)$ is a complete metric space, and $w: X \rightarrow X$ is a compression map on $X$. Then, $W$ has a unique fixed point $x_{w}$ of $W(x)=x_{w}$, For any $x \in X$, the sequence $\left\{W^{n}(x): n=1,2, \ldots\right\}$ converges to $X_{w}$.

Theorem 2 shows that the compact family in the complete metric space must have fixed points. Theorem 2 is the general application of theorem 1 . It is the theoretical basis and foundation for the affine stability of fractal images.

Theorem 3: If $X: w_{n}, n=1,2, \ldots, N$ is the iteration function of the complete metric space $(X, d)$, the compression ratio is $c$, Next, the transform $W: F(X) \rightarrow F(X)$ is defined as follows:

$$
W(B)=\cup_{n=1}^{N} w_{n}(B)
$$

Where $B \in F(X)$. Therefore, $W$ is a compression mapping of the $(F(X), d)$ compression ratio $c$. The only fixed point is $d(W(B), W(B)) \leq c d(B, C), B, C \in F(X)$. It satisfies the following formula: 


$$
A=W(A)=\underset{n=1}{\cup} w_{n}^{N}(A)
$$

Furthermore, for any $A \in F(X), A=\lim _{n \rightarrow \infty} W^{n}(B)$.

The fixed point or fixed set (set or point A) in Theorem 3 is called the attractor of this iterated function system. The attractors of the iterated function system are generally fractal or have basic properties of fractal. It is called deterministic fractal.

Theorem 4: (Collage theorem) Suppose that the metric space $(X, d)$ is complete, and for a certain compression factor that is $s(0 \leq 1)$, the iteration function series with the fixed set $A$ is $X$ : $w_{n}, n=1,2, \ldots, N$. This means that the following holds

$$
h_{d}\left(L, W_{n=1}^{N}(L)\right) \leq \varepsilon,(L \in H(X))
$$

In the formula, $h_{d}$ is the Hausdorff measure, and $H(X)$ is the mapping. Thus, the iterative function system $W$ is

$$
h_{d}(L, A) \leq(1-s)^{-1} h_{d}\left(L, W_{n=1}^{N}(L)\right),(L \in H(X))
$$

As one of the important theorems in fractal geometry - the collage theorem, it illustrates the degree of self-similarity between a set and its corresponding invariant set in fractal geometry. For an iteration function $X: w_{n}, n=1,2, \ldots, N$, its attractor $A$ is approximately equal to a given set $L$. If we find a set of squeezed transformations that satisfy the collage theorem, the given set $L$ can be approximated by using the attractor of this set of compression transformations.

\subsection{Fractal interpolation function}

Definition 9: If the function $f$ is an interpolation function, it satisfies $\left\{\left(x_{i}, F_{i}\right) \in R^{2}: i=1,2, \mathrm{~L}, \mathrm{~N}\right\}$, $x_{1}<x_{2}<x_{3}<x_{N}$, and $f$ is the continuous function $f:\left[x_{1}, x_{N}\right] \rightarrow R$.

Theorem 5: Suppose that $a \leq x_{1}<x_{2}<x_{3}<\cdots<x_{N} \leq b$. Thus, the Nth order polynomial $f_{N}(x)$ that satisfies the interpolation condition is unique.

The traditional interpolation algorithm can be divided into various methods according to the constructed interpolation function, such as polynomial interpolation, spline interpolation, cubic spline interpolation, and B-spline interpolation. The commonly used interpolation algorithm can be divided into Newton interpolation according to function, Lagrange interpolation and so on. According to Theorem 5, it can be ensured that for the same data, the polynomial satisfying the interpolation condition regardless of which interpolation algorithm is used is the same polynomial, but the expression form will be different.

For a planar data set $\left\{\left(x_{i}, y_{i}\right) \in R^{2}: i=1,2, \ldots, N\right\}$, construct an iterative function on $R^{2}$ such that its attractor is a given data set $\left\{\left(x_{i}, y_{i}\right) \in R^{2}: i=1,2, \ldots, N\right\}$.

Assuming that the data set on $R^{2}$ is $\left\{\left(x_{i}, y_{i}\right) \in R^{2}: i=1,2, L, N\right\}, f:\left[x_{1}, x_{N}\right] \rightarrow R$ is a continuous function of the interpolation and the data set, The form of the straight line in the interval $\left[x_{i-1}, X_{i}\right](i=1,2, L, N)$ is as follows

$$
f(x)=y_{i-1}+\frac{y_{i}-y_{i-1}}{x_{i}-x_{i-1}}\left(x-x_{i-1}\right)
$$

Where $x \in\left[x_{i-1}, x_{i}\right](i=1,2, L, N)$

Let this function $f(x)$ be a piecewise linear function, as shown in Fig. 1. These $f(x)$ changes to an iterative function, which is then interpolated, and the functional sequence is iterated over 
the data. Thus, the function sequence $\left\{f_{n+1}(x)=\left(T f_{n}\right)(x)\right\}_{n=0}^{\infty}$ will converge to a fixed point, and a fractal image.

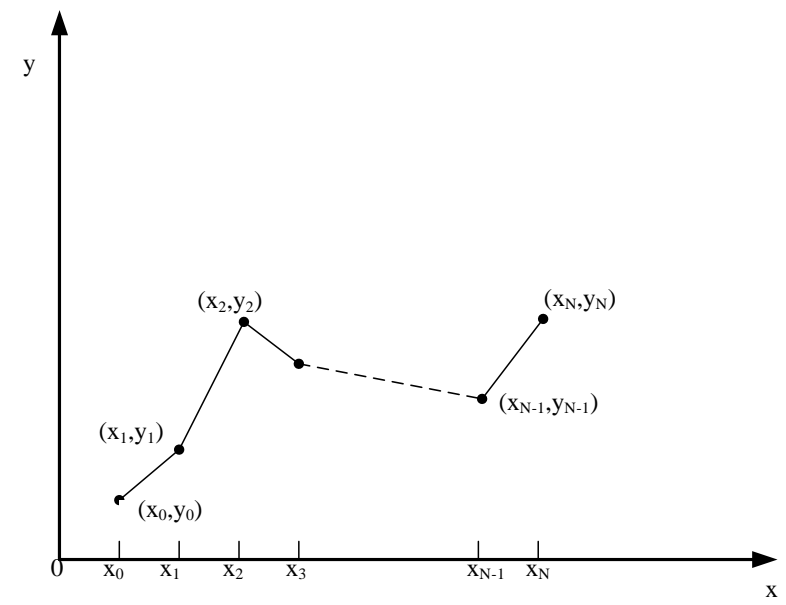

Fig. 1. Piecewise linear function

By interpreting the graph of Fig. 1, it can get a fractal curve. It is the attractor $G$ of $\operatorname{IFS}\left\{R^{2}\right.$ : $\left.W_{1}, W_{2}, \ldots, W_{N}\right\}$

$$
W_{n}\left[\begin{array}{l}
x \\
y
\end{array}\right]=\left[\begin{array}{ll}
a_{n} & 0 \\
c_{n} & 1
\end{array}\right]\left[\begin{array}{l}
x \\
y
\end{array}\right]+\left[\begin{array}{l}
e_{n} \\
f_{n}
\end{array}\right], \quad n=1,2, \cdots, N
$$

Let $l=x_{N}-\chi_{0}$ such that

$$
\begin{aligned}
& a_{n}=\frac{1}{l}\left(x_{n}-x_{n-1}\right) \\
& c_{n}=\frac{1}{l}\left(y_{n}-y_{n-1}\right) \\
& e_{n}=\frac{1}{l}\left(x_{N} x_{n-1}-x_{0} x_{n}\right) \\
& f_{n}=\frac{1}{l}\left(x_{N} y_{n-1}-x_{0} y_{n}\right)
\end{aligned}
$$

At this time, $\mathrm{G}$ is a non-empty compact set on $\mathrm{R} 2$ that satisfies the following formula:

$$
G=\bigcup_{n=1}^{N} W_{n}(G)
$$

It can be seen from this example that IFS can be used as an interpolation transformation, but fractal interpolation is not an ordinary data interpolation, and its interpolated curve must be a fractal curve. In other words, any part of the interpolation curve must be a differentiable curve.

This requirement can be met by analyzing its self-similarity and any iteration of $\bigcup_{n=1}^{N} W_{n}$.

Assuming that the functional sequence $\left\{f_{n+1}(x)=\left(T f_{n}\right)(x)\right\}_{n=0}^{\infty}$ converges to a fixed point on the map $T$, Fig. 2 shows the basic principle of the fractal interpolation. In the figure $A, B$, and $C$ are the three points of the interpolation data. If $f_{0}(x)$ is the line segment $A C$, then $f_{1}(x)$ is the segmentation function of the two segments, $f_{2}(x)$ is the four lines of the contour ADBEC, and $f_{3}(x)$ are the eight segments of the second row of the left contour. In addition, $\triangle A D B$, and $\triangle \mathrm{BEC}$ have similarity to the original $\triangle \mathrm{ABC}$, and a series of double triangles obtained after each iteration have similarity to the original triangle. The line $A B C$ is a two-line segment, and 
its two affine changes $W_{1}$ and $W_{2}$ respectively map the lines $A B$ and $B C$, and the resulting segments are $W_{1}(A B)=A D, W_{2}(A B)=D B$ and $W_{2}(B C)=E C$. Suppose that $W=W_{1} \cup W_{2}$. Thus, $\lim _{n \rightarrow \infty} W^{n}(A B C)$ is the outline of the curve in the Fig. 2.

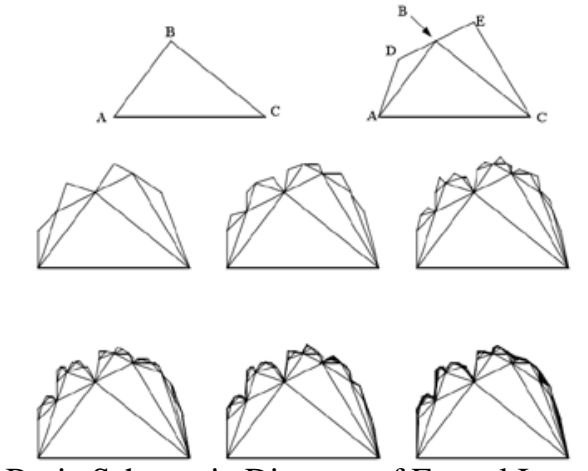

Fig. 2. Basic Schematic Diagram of Fractal Interpolation

\section{Bi-dimensional Empirical Mode Decomposition Interpolation Algorithm Based on Fractal Theory}

\subsection{One-dimensional Brownian motion}

Brownian motion is a random motion, Brownian motion is a continuous collision of many particles. Adjacent particles lead to the particle's movement direction of the continuous change of the movement process, and the change trajectory is an irregular curve. The one-dimensional Brownian motion can be described by a random process as follows: For any $t_{1}$ and $t_{2}[23,24]$

$$
\begin{aligned}
& L_{H}\left(t_{1}\right)-L_{H}\left(t_{2}\right) \text { according to the Gaussian distribution (11) } \\
& E\left(\left|L_{H}\left(t_{2}\right)-L_{H}\left(t_{1}\right)\right|^{2}\right) \infty\left|t_{2}-t_{1}\right|
\end{aligned}
$$

For either $t_{0}$ or $\gamma>0, L\left(t_{0}+t\right)-L\left(t_{0}\right)$ and $\frac{1}{\sqrt{\gamma}}\left(L\left(t_{0}+\gamma t\right)-L\left(t_{0}\right)\right)$ have the same joint distribution function. In other words, the increment of $\mathrm{L}$ has a self-similar characteristic according to the statistical law.

\subsection{Fractal Brown Function}

Formula (12) can be adjusted to the following formula

$$
\operatorname{Var}\left(L\left(t_{2}\right)-L\left(t_{1}\right)\right) \infty|\Delta t|^{2 H}
$$

Where Var is the formula and $L(t)$ is the one-dimensional fractal Brownian function. $H$ changes from the original $H=1 / 2$ to $0<H<1$, which indicates the degree of irregularity of $L(t)$.

The fractal Brownian motion is a continuous function. Let $t \in R^{n}$, and $L(t)$ be a real-valued stochastic function with respect to $t$. If there exists a constant $H(0<H<1)$.

$$
\operatorname{Pr}\left\{\frac{L_{H}(t+\Delta t)-L_{H}(t)}{\|\Delta t\|^{H}}<x\right\}=F(x)
$$

If $F(x)$ is independent of $\Delta t, t$, then $L(t)$ is a fractal Brownian motion. $T$ represents a point in the $R^{n}$ space, $\Delta t$ represents the offset of the point, $\|\Delta t\|$ represents the Euclidean distance, $F(x)$ represents the Gaussian random distribution function, and the fractal dimension is defined by 
$L_{H}(t)$

$$
D=E+1-H
$$

In formula (15), $\mathrm{D}$ represents the topological dimension.

$L_{H}(t)$ has the following properties [23, 24]:

$$
E\left[L_{H}(t+\Delta t)-L_{H}(t)\right]^{2}=E\left|L_{H}(t+1)-L_{H}(t)\right|^{2}\|\Delta t\|^{2 H}
$$

Under normal circumstances, the average fractal dimension of the graphic can be obtained by the following formula:

$$
D=3-H
$$

$H$ represents a fractal self-similar parameter, which has the following characteristics [23, 24]:

(1) It is normal Brownian motion when $H=1 / 2$;

(2) $D=N+1$ when $H=0$, which represents the ideal fractal Brownian motion;

(3) $D=N$ when $H=1$, which is a normal set that can be described by the common set language;

(4) When $0<H<1$, If $H$ is smaller, $L(t)$ vibrates more intensely, and the resulting image has more complex changes. When the value of $H$ is larger, the vibration of $L(t)$ is less violent, and the change of the image obtained by it is simpler. Therefore, $H$ is a quantitative indicator of the complexity or roughness of the image surface.

If the zero-mean Gaussian random distribution function is represented by $L(t)$, then its specific form can be expressed by the following formula

$$
F(x)=2 \int_{-\infty}^{0} \frac{1}{\sqrt{2 \pi} \sigma} \exp \left(\frac{-s^{2}}{2 \sigma^{2}}\right) d s
$$

According to formula (14), we can know that

$$
E\left|\frac{L(t+\Delta t)-L(t)}{\|\Delta t\|^{H}}\right|=E|x|=2 \int_{0}^{+\infty} x \frac{1}{\sqrt{2 \pi} \sigma} \exp \left(\frac{-x^{2}}{2 \sigma^{2}}\right) d x=\frac{2 \sigma}{\sqrt{2 \pi}}=C
$$

Thus, formula (19) can be expressed by the following formula

$$
\log E|L(t+\Delta t)-L(t)|-H \log \|\Delta t\|=\log C
$$

Because $H$ and $C$ are constants, we know that $\log E|L(t+\Delta t)-L(t)|$ and $\log \|\Delta t\|$ have a linear relationship through formula (20). In Cartesian coordinates, $(\log \|\Delta t\|, \log E|L(t+\Delta t)-L(t)|)$ represents the slope of the line, which is represented by $H$, and it fits a straight line through this information to obtain the value of $H$.

\subsection{Fractal Features of Images}

The general image fractal feature is to use the method of Section 2.2 fractal Brown function to analyze the specific image to get its distribution function and fractal dimension, as follows.

(1) Distribution function

One of the characterizations of the image surface shape is its distribution function $F(x)$. In this paper, $F(x)$ is the zero-mean Gaussian distribution function $N\left(0, \sigma^{2}\right)$, whose characteristic is determined $b y \sigma^{2}$. Here by formula (18) and formula (19) obtained.

(2) Fractal dimension

The fractal dimension is a fractal description of the image characteristic parameters. It fractals the characteristics of the image to be processed through Section 2.3, and its fractal dimension is obtained using formula (15). Under the same distribution function $F(x)$, the 
bigger the image fractal dimension $D$ is, the more the object under test is rougher and vice versa.

\subsection{Random midpoint displacement method}

The fractal theory can be used to obtain a specific stochastic mid-point displacement method, which expresses the value of $\left(x_{m i}, y_{m i}\right)$ using the following formula. Specifically [23, 24]

$$
\begin{aligned}
& x_{m i}=\left(x_{i}+x_{i+1}\right) / 2+s \cdot w \cdot \operatorname{rand}() \\
& y_{m i}=\left(y_{i}+y_{i+1}\right) / 2+s \cdot w \cdot \operatorname{rand}()
\end{aligned}
$$

In Eq. (21) and Eq. (22), $x$ is the horizontal axis and $y$ is the vertical axis. $s$ and $w$ are control parameters, $s$ controls the left and right direction of $x$ movement and the up and down direction of $y$ movement, $w$ is the actual movement distance of $s$ in the $x$ and $y$ directions, and rand( ) controls the moving random variables.

The random variable $s \cdot w \cdot r a n d()$ is represented by the normal random function $\operatorname{stdev} * N(0,1)$ such that $s \cdot \operatorname{rand}()$ is a normal distribution. The standard deviation evolution law of the new interval can be recorded by parameter $H$. According to B. B. Mandelbrot's theory, its dimension is $1+H$.

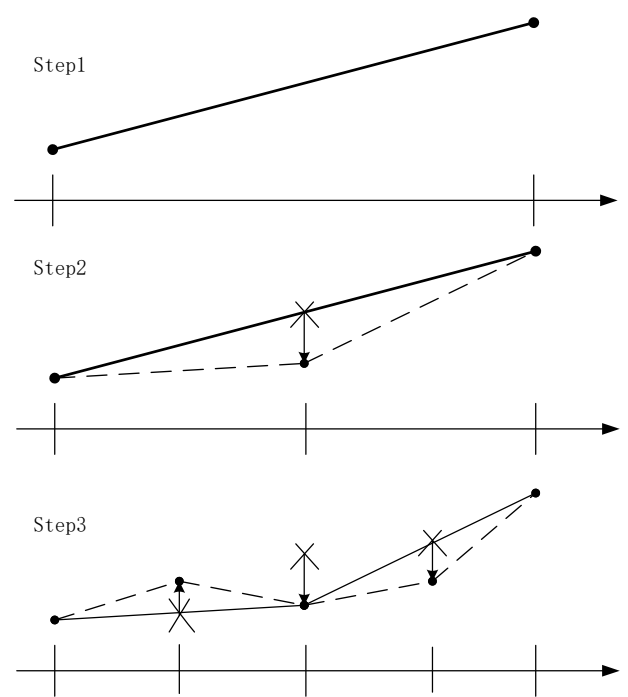

Fig. 3. Random midpoint displacement method diagram

The idea of the one-dimensional random midpoint displacement method is as follows. First, the two endpoints of the existing endpoint attribute line's midpoint is used as the mean value of both ends and any random displacement. Next, the two line segments after displacement are repeated, until the given condition is reached, As shown in Fig. 3, the line's two-dimensional promotion is similar to its one-dimensional promotion.

\subsection{Bi-dimensional Empirical Mode Decomposition Interpolation Algorithm Based on Fractal Theory}

Fractal interpolation algorithms includes the following. First is image feature extraction, and second is the image interpolation. The details are as follows.

(1) Image feature extraction

1) Describe $E\left|L_{H}(t+\Delta t)-L_{H}(t)\right|^{2}$ by calculating the expected value of the pixel brightness difference in the spatial distance of the image $\Delta t$. 
2) Determine the scale limit parameters $|\Delta t|_{\min }$ and $|\Delta t|_{\max }$.

If the image is an ideal fractal feature, then its fractal dimension is constant. However, the actual image may not be completely perfectly fractal. Therefore, it needs to determine a scale range, which ensures that the fractal dimension in this range is a constant. The specific determination method is as follows. Draw a fractal dimension diagram, which is $\log E\left|L_{H}(t+\Delta t)-L_{H}(t)\right|^{2}$ relative to the $\log |\Delta t|$ curve, wherein the upper and lower limits of the straight line segments are $|\Delta t|_{\min }$ and $|\Delta t|_{\max }$.

3) Calculate the standard deviation $\delta$ of the parameter $H$ and the pixel gray level normal distribution, and get the following relationship according to formula (16)

$$
\log E\left|L_{H}(t+\Delta t)-L_{H}(t)\right|^{2}-2 H \log |\Delta t|=\log \sigma^{2}
$$

In the formula, $\sigma^{2}=E\left|L_{H}(t+1)-L_{H}(t)\right|^{2} . H$ and $\sigma$ can be obtained by solving the above formula.

(2) Bi-dimensional empirical mode decomposition fractal interpolation

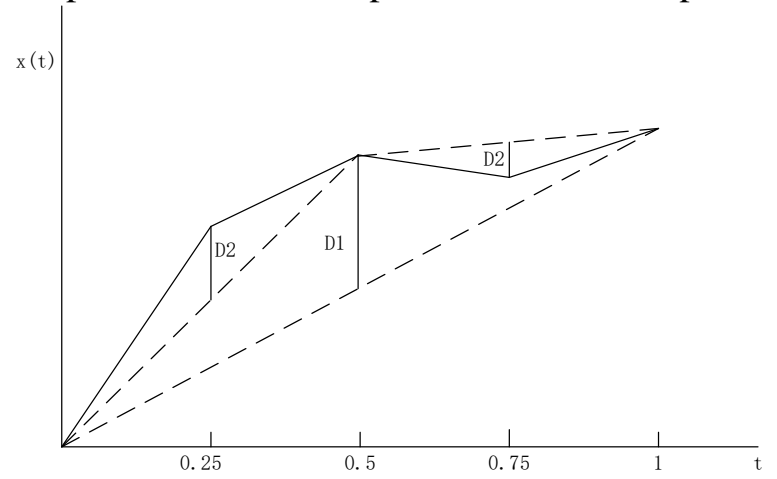

(a)

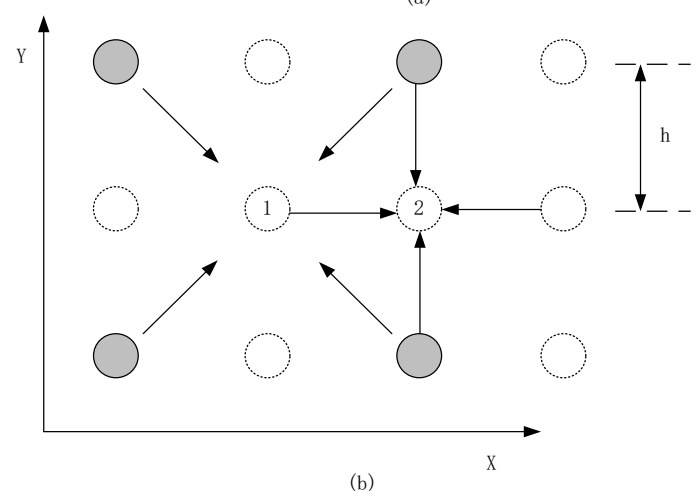

Fig. 4. Random midpoint shift recursive method

The bi-dimensional empirical mode decomposition fractal interpolation algorithm is essentially the process of the recursive random midpoint displacement method, as shown in Fig. 4, which is achieved by the following formula, for the pixels in the image $(i, j)$. Assuming $i, j$, when all are odd numbers, its gray value $L_{H}$ has been determined, and when $i$ and $j$ are both even numbers, it can be obtained as follows.

$$
\begin{aligned}
& L_{H}(i, j)=\frac{1}{4}\left\{L_{H}(i-1, j-1)+L_{H}(i+1, j-1)+L_{H}(i+1, j+1)\right. \\
& \left.+L_{H}(i-1, j+1)\right\}+\sqrt{1-2^{2 H-2}}\|\Delta t\| \cdot H \cdot \sigma \cdot G
\end{aligned}
$$

When $i, j$, there is only one, even when 


$$
\begin{aligned}
& L_{H}(i, j)=\frac{1}{4}\left\{L_{H}(i, j-1)+L_{H}(i-1, j)+L_{H}(i+1, j)\right. \\
& \left.+L_{H}(i, j+1)\right\}+2^{-H / 2} \sqrt{1-2^{2 H-2}}\|\Delta t\| \cdot H \cdot \sigma \cdot G
\end{aligned}
$$

Where $G$ is a Gaussian random variable with an $N(0,1)$ ) distribution and $\|\Delta\|$ is the inter-sample distance. Therefore, it can use the $H$ and $\sigma$ of the original image's feature description information to get the interpolation point's brightness.

This approach reaches the set spatial resolution and continues to re-start the above steps. In each iteration process, it needs to insert the midpoint, which is a Gaussian random variable, and the expectation is the average of four adjacent points. The offset of the point can be jointly determined by $H$ and $\sigma$, which can describe the image's characteristic information. When $H=0$, the offset of this point relative to the average of four adjacent points needs to be determined by $\sigma$. When $H=1$ and the variance is 0 , the average of the four adjacent points is equivalent to the linear interpolation. If the value of is constant, the smaller that the value of $H$ is the more random that the interpolation point will be for the specific interpolation principle that is shown in Fig. 5.

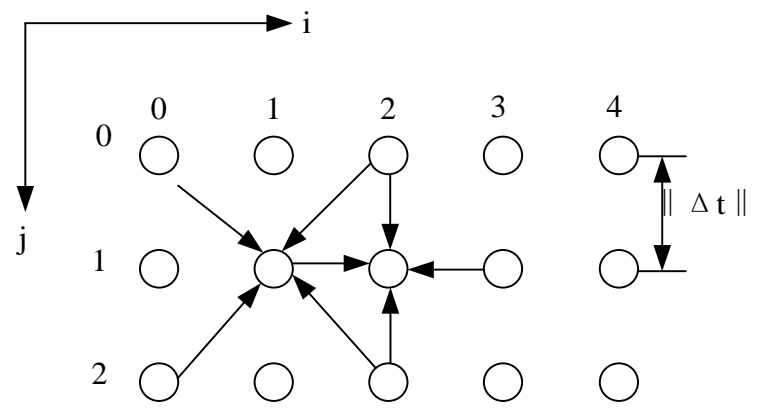

Fig. 5. Fractal interpolation basic diagram

The image under testing has a very high self-similarity characteristic in everyday production and life, and the fractal interpolation is used to reverse the self-similarity. Therefore, this is also the most important reason that the fractal interpolation algorithm can obtain a better interpolation effect.

\subsection{Bi-dimensional empirical mode decomposition algorithm interpolation of the concrete realization of the process}

Since bi-dimensional empirical mode decomposition algorithm interpolation can be achieved by the above principles, the related formulas and matlab programming, the main steps are:

(1) Read in the picture $I$, and it is transformed into $M \times N$ data matrix;

(2) For the $I(i, j)$ matrices, $i$ and $j$ are all even numbers and are interpolated by the formula

(3) Interpolate with (33) for $I(i, j)$ with only one even number;

(4) Perform a data rounding operation on the $I(i, j)$ matrix obtained through step (2) and the step (3);

(5) The matrix $I(i, j)$ is converted into a picture output.

\section{Fractal interpolation parameter particle swarm optimization}

To further improve the computational efficiency of the fractal interpolation algorithm, the particle swarm optimization is performed on the parameters such as the vertical scale factor 
and the initialization involved in the fractal interpolation algorithm. The specific procedure is as follows [25-33].

We use $X_{i}=\left(x_{i 1}, x_{i 2}, \ldots, x_{i D}\right)$ denoting the $i$ th particle, its best position is represented by $p_{\text {best }}$ which is expressed in term of $P=\left(p_{i 1}, p_{i 2}, \ldots, p_{i D}\right)$, and its velocity in term of $V_{i}=\left(v_{i 1}, v_{i 2}, \ldots, v_{i D}\right)$. The best place for the entire group is represented by $g_{\text {best }}$. The $d$-th dimension $(1 \leq d \leq D)$ of each generation of each particle is dealt with specifically by the relevant method as follows:

The particle swarm optimization algorithms that are used to optimize the parameters involved in the basic steps are as follows:

$$
\begin{gathered}
v_{i d}=w v_{i d}+c_{1} \operatorname{rand}()\left(p_{i d}-x_{i d}\right)+c_{2} \operatorname{Rand}()\left(p_{g d}-x_{i d}\right) \\
x_{i d}=x_{i d}+v_{i d}
\end{gathered}
$$

Step 1: Population initialization, where $n$ particles are assumed, the position and velocity is represented by $x_{i}^{0}$ and $v_{i}^{0}$, respectively, and the number of iterations is set;

Step 2: Calculate the fitness of all particles in a certain state, denoted as $p_{i}$;

Step 3: Compare the fitness value $p_{i}$ calculated in step 2 with the optimal solution $p_{\text {best }_{i}}$ obtained from the optimization, if $p_{i}<p_{\text {best }_{i}}$, the new fitness value is replaced by the new fitness value and the new one is replaced by the new one Of the particles to replace the particles of the previous stage, that is $p_{\text {best }_{i}}=p_{i}$ and $x_{\text {best }_{i}}=x_{\text {best }_{i}}$.

Step 4: By comparing the optimal fitness value $p_{\text {best }_{i}}$ of each particle in the population with the optimal fitness value $g_{\text {best }_{i}}$ of all particles, if $p_{\text {best }_{i}}<g_{\text {best }_{i}}, p_{\text {best }_{i}}$ of the particle is substituted for a of all particles $g_{\text {best }}$, and the position and state of the particle save, ie $g_{\text {best }_{i}}=p_{\text {best }_{i}}$ and $x_{\text {best }_{i}}=x_{\text {best }_{i}}$;

Step 5: Through the calculation of step 1 to step 4, we can get the new velocity and position of the particle, and use it to replace the corresponding value of the original particle to generate new particle.

Step 6: If the setting optimization condition has not been reached through the operation of step 1 to step 5 , then step 2 is performed again until the setting condition is satisfied. The specific optimization calculation process is shown in Fig. 6.

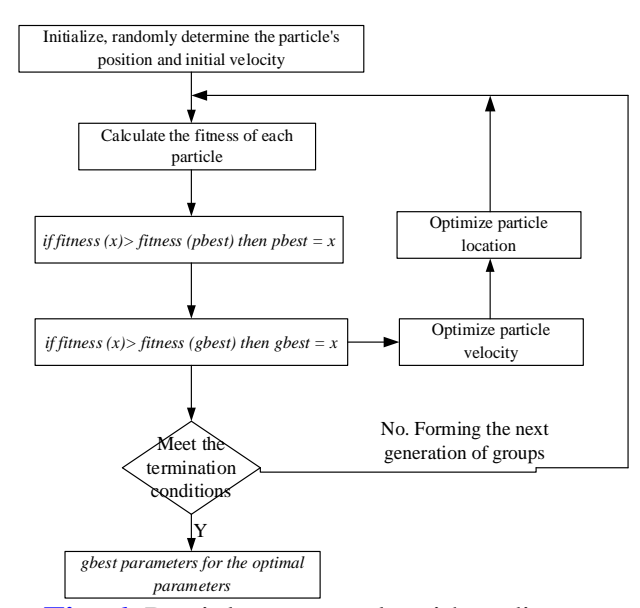

Fig. 6. Particle swarm algorithm diagram 


\section{Image Fractal Interpolation Experimental Analysis}

\subsection{Image quality evaluation}

The ultimate goal of image processing is to obtain a clearer image in order, to achieve the purpose of changing the visual effects. Therefore, it is very important to evaluate the quality of the processed images. The current image quality evaluation is based on two ways as: subjective evaluation and objective evaluation. The following three types of image quality evaluation are adopted as the basic characteristics and application scenarios for analysis and description.

(1) Subjective evaluation of image quality

The subjective evaluation of image quality is similar to a diving competition in sports. The image quality is scored by multiple referees, and then the competition's results are determined according to the rules. The referee who subjectively evaluates the image quality may be a general practitioner or a professionally trained person.

The subjective evaluation of the image quality is subjective and contains more content. The evaluation results can be easily disturbed by the evaluators. The evaluation results obtained cannot fully reflect the real quality of the images.

(2) Objective evaluation of image quality

This is the objective evaluation of image quality through the PSNR, MSE and other indicators of image quality before and after the judging. It involves with the specific meanings of MSE and PSNR and see [23, 35].

(3) Wang et al. proposed an approach to image fidelity measurement, which may also prove highly effective for measuring thefidelity of other signals, such as the structural similarity (SSIM) index. It involves with the specific meanings of SSIM (see [34, 35]).

Through the above analysis, we can know that the objective evaluation of the image quality can better reflect the quality of the processed image. We will use this method to evaluate the image quality interpolated by the interpolation algorithm proposed in this paper, so as to objectively verify the performance and advantages of the interpolation algorithm proposed here.

\subsection{Experiment 1}

To verify the advantages of the image interpolation algorithm proposed in this paper, we choose Lena and Couple from standard image testing library. The experimental images are shown in Fig.7. In the experiment, the two images are reduced by 2 times, 4 times, 8 times and 16 times respectively, and then the sizes of the two images are restored by using the interpolation algorithm, double three times, double non-linearity and the nearest neighbor interpolation, respectively. The reduction effect is shown respectively in Fig. 8 to Fig. 15. The PSNR, MSE and calculation time are shown in Tables 1-3.

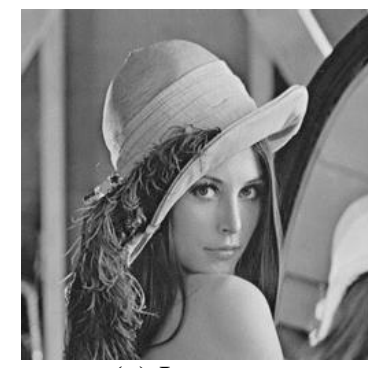

(a) Lena

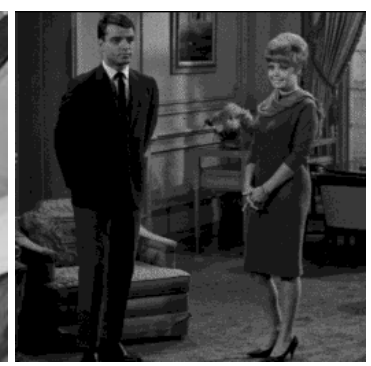

(b) Couple

Fig. 7. Lena and Couple experimental images 


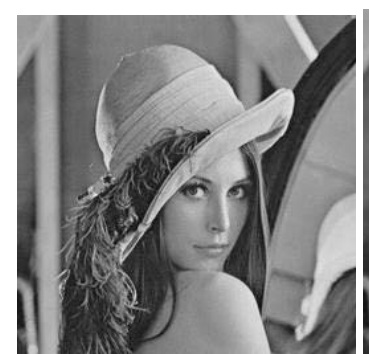

(a)

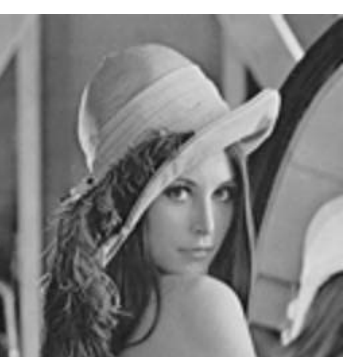

(b)

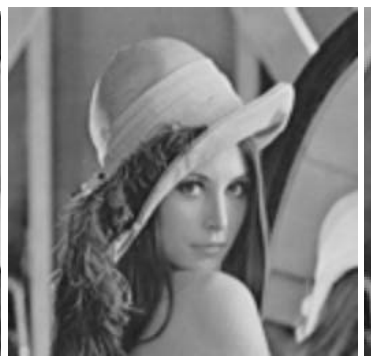

(c)

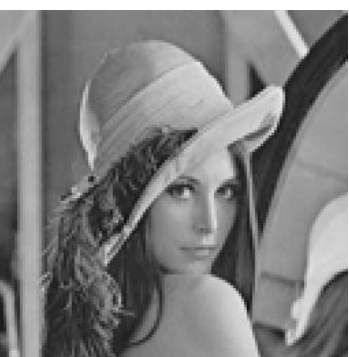

(d)

Fig. 8. Lena image reduced by the 2 times different algorithm interpolation zoom to the original sized renderings((a) Fractal interpolation, (b) Bicubic interpolation, (c) Double nonlinear interpolation, and (d) Nearestneighbor interpolation)

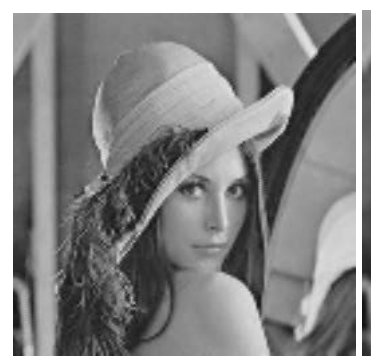

(a)

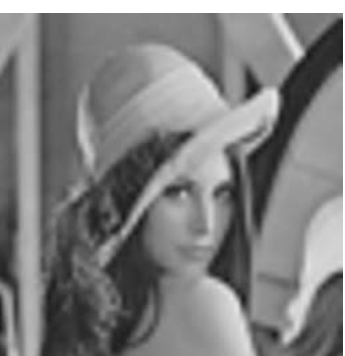

(b)

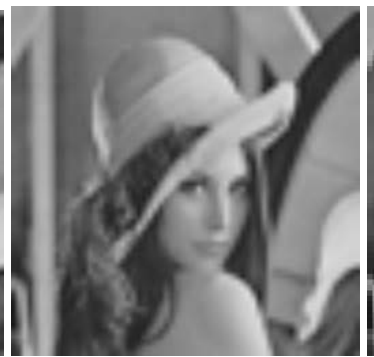

(c)

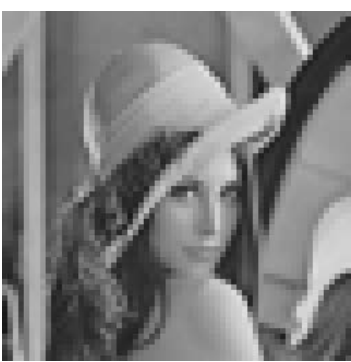

(d)

Fig. 9. Lena image reduced by the 4 times different algorithm interpolation zoom to the original sized renderings((a) Fractal interpolation, (b) Bicubic interpolation, (c) Double nonlinear interpolation, and (d) Nearestneighbor interpolation)

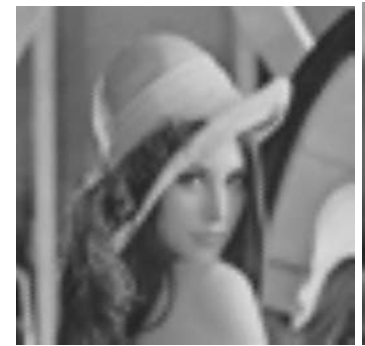

(a)

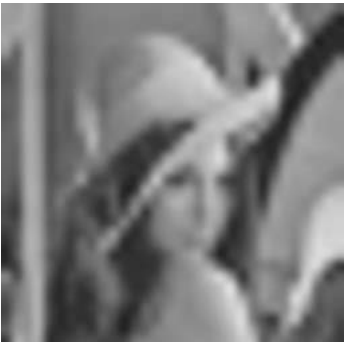

(b)

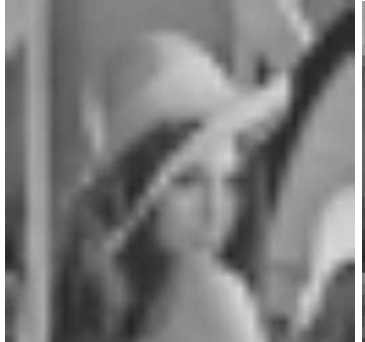

(c)

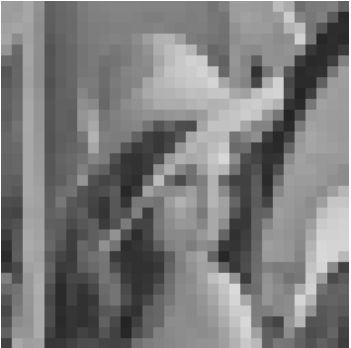

(d)

Fig. 10. Lena image reduced by the 8 times different algorithm interpolation zoom to the original sized renderings((a) Fractal interpolation, (b) Bicubic interpolation, (c) Double nonlinear interpolation, and

(d) Nearestneighbor interpolation)

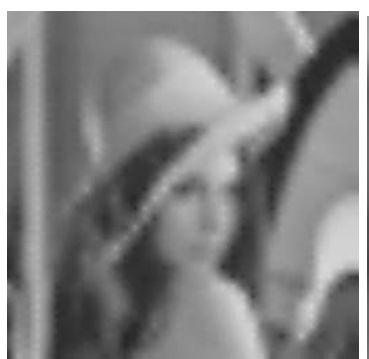

(a)

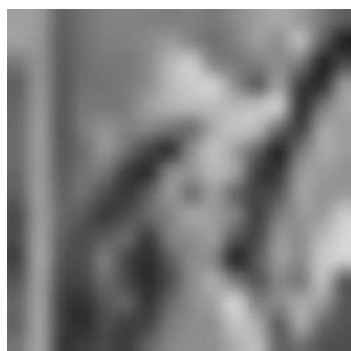

(b)

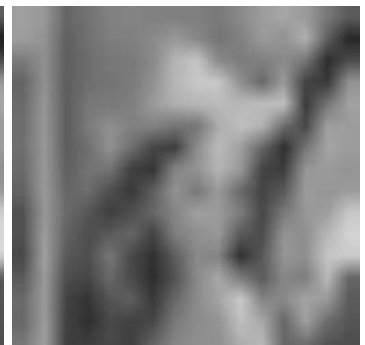

(c)

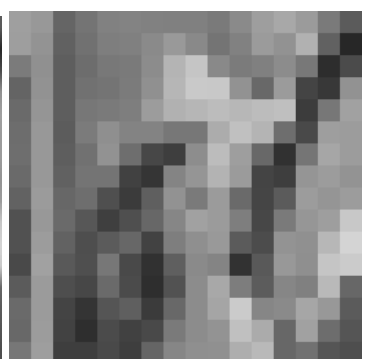

(d)

Fig. 11. Lena image reduced by the 16 times different algorithm interpolation zoom to the original sized renderings((a) Fractal interpolation, (b) Bicubic interpolation, (c) Double nonlinear interpolation, and (d) Nearestneighbor interpolation) 


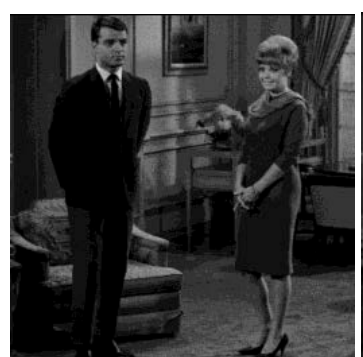

(a)

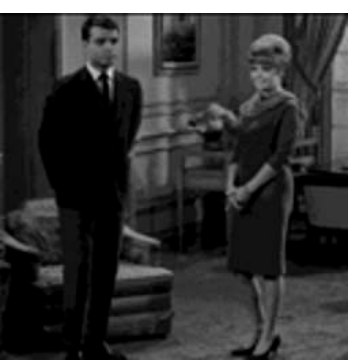

(b)

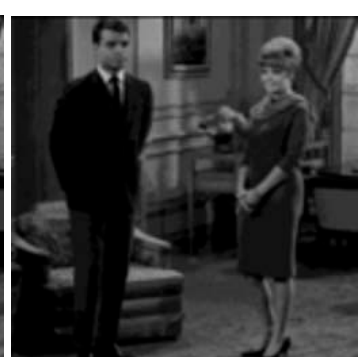

(c)

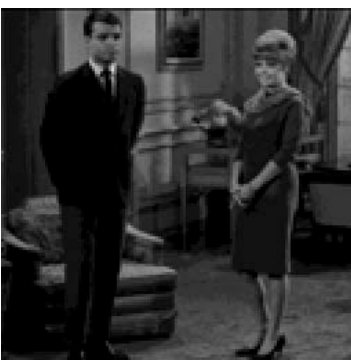

(d)

Fig. 12. Couple image reduced by the 2 times different algorithm interpolation zoom to the original sized renderings

((a) Fractal interpolation, (b) Bicubic interpolation, (c) Double nonlinear interpolation, and (d)

Nearestneighbor interpolation)

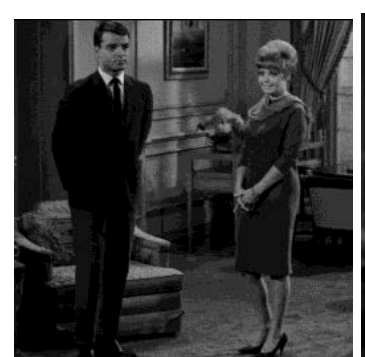

(a)

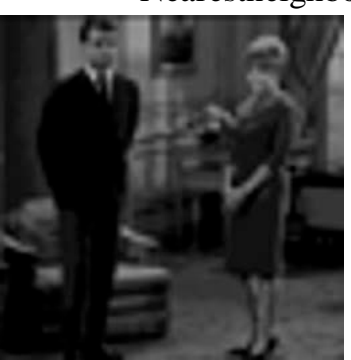

(b)

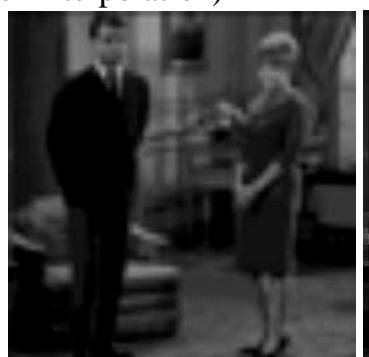

(c)

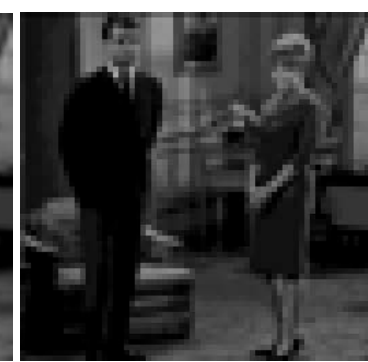

(d)

Fig. 13. Couple image reduced by the 4 times different algorithm interpolation zoom to the original sized renderings((a) Fractal interpolation (b) Bicubic interpolation (c) Double nonlinear interpolation (d) Nearestneighbor interpolation)

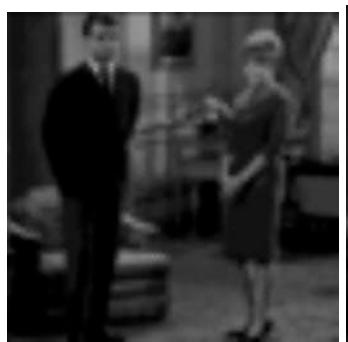

(a)

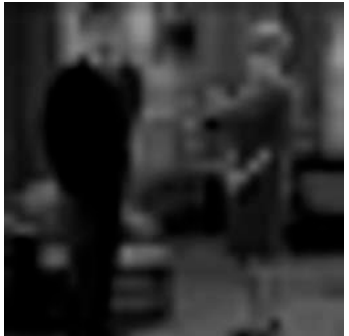

(b)

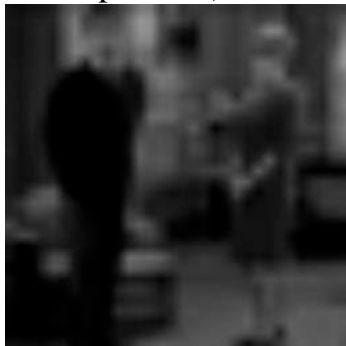

(c)

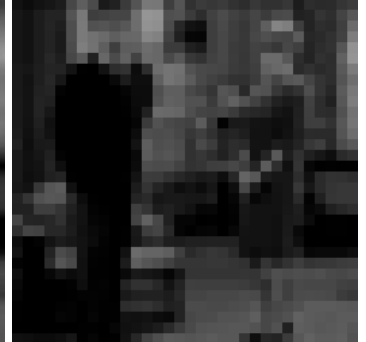

(d)

Fig. 14. Couple image reduced by the 8 times different algorithm interpolation zoom to the original sized renderings((a) Fractal interpolation, (b) Bicubic interpolation, (c) Double nonlinear interpolation, and (d) Nearestneighbor interpolation)

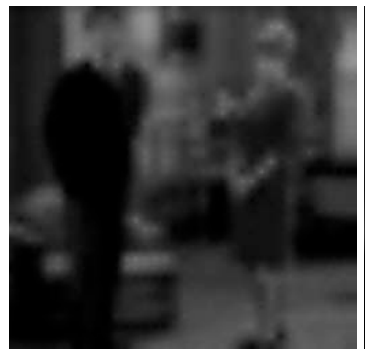

(a)

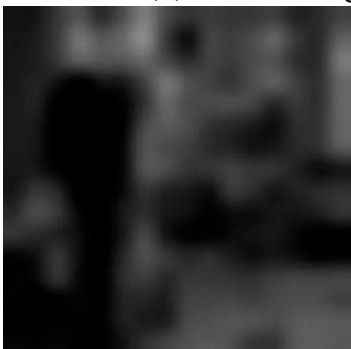

(b)

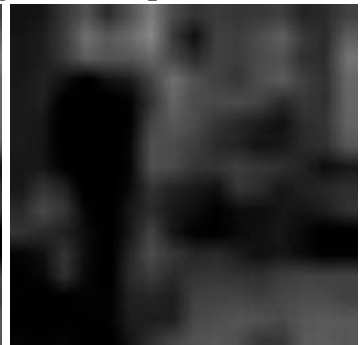

(c)

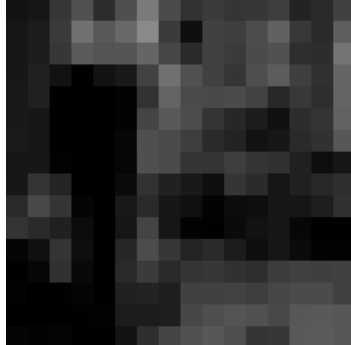

(d)

Fig. 15. Couple image reduced by the 16 times different algorithm interpolation zoom to the original sized renderings((a) Fractal interpolation, (b) Bicubic interpolation, (c) Double nonlinear interpolation, and (d) Nearestneighbor interpolation) 
It can be seen from Fig. 8 to Fig. 11 that the Lena image is magnified to its original size by different interpolation algorithms after being reduced by 2, 4, 8 and 16 times, respectively. In this paper, the proposed effect after interpolation is 8 and 16 times Obviously better than the other three interpolation algorithms; and especially in the case of 16 times, the effect of the interpolation algorithm in this paper is still relatively clear, the other three algorithms have been relatively vague renderings, especially the nearest neighbor interpolation The resulting graph is unable to distinguish the image of the character information, therefore, the advantage of the algorithm is obvious. In the cases of 2 and 4 times, the algorithm in this paper also has advantages, but the subjective visual perception is not obvious. Follow-up will be analyzed in detail through objective indicators such as PSNR and MSE.

Couple images are reduced by 2, 4, 8 and 16 times, respectively, and then magnified to the original size by different interpolation algorithms. As shown in Fig. 12 to Fig. 15, we can see that the effect of interpolation after 8 and 16 times is obviously better than the other three algorithms. In the case of 16 times, the interpolation result image obtained in this paper can basically reflect the overall information of the original image, and the results obtained by the other three algorithms are not complete. The original image information is summarized, which directly proves the advantages of the interpolation algorithm in this paper. In 2 and 4 times cases, the algorithm also has advantages, but subjective visual perception is not obvious, the paper will be followed by specific indicators of comparative analysis.

It can be seen from Table 1 that after the 2, 4, 8, and 16 times of reduction, it is enlarged to its original size. The interpolation algorithm proposed in this paper has the highest PSNR, which objectively shows that the interpolation algorithm in this paper has the uniqueness of the interpolation, and the interpolation effect is the best. Especially in the case of 8,16 times, the other three kinds of interpolation algorithm has appeared more vague circumstances, and the proposed interpolation algorithm restore the original size of the image, not only the image is more clearly, the PSNR of the image restored to its original size is also the highest among several interpolation algorithms. It proves that the interpolation algorithm proposed in this paper has the highest image quality.

Table 1. PSNR (unit: dB) statistics for different algorithms for different magnifications

\begin{tabular}{cccccc}
\hline $\begin{array}{c}\text { Experimental } \\
\text { images }\end{array}$ & Gain & $\begin{array}{c}\text { This paper } \\
\text { method }\end{array}$ & $\begin{array}{c}\text { Double } \\
\text { three times } \\
\text { algorithm }\end{array}$ & $\begin{array}{c}\text { Double } \\
\text { non-linear } \\
\text { algorithm }\end{array}$ & $\begin{array}{c}\text { Nearest } \\
\text { neighbor } \\
\text { algorithm }\end{array}$ \\
\hline \multirow{2}{*}{ Lena } & 2 & 31.79 & 30.08 & 28.91 & 28.26 \\
& 4 & 26.63 & 25.62 & 24.96 & 24.03 \\
& 8 & 22.26 & 22.23 & 21.77 & 21.13 \\
Couple & 16 & 20.07 & 19.70 & 19.27 & 19.02 \\
& 2 & 38.75 & 30.42 & 29.62 & 28.88 \\
& 4 & 26.67 & 26.65 & 26.14 & 25.53 \\
& 8 & 24.27 & 23.74 & 23.42 & 23.00 \\
\hline \hline
\end{tabular}

Table 2. MSE statistics for different algorithms for different magnifications

\begin{tabular}{cccccc}
\hline \hline $\begin{array}{c}\text { Experimental } \\
\text { images }\end{array}$ & Gain & $\begin{array}{c}\text { This } \\
\text { paper's } \\
\text { method }\end{array}$ & $\begin{array}{c}\text { Double } \\
\text { three times } \\
\text { algorithm }\end{array}$ & $\begin{array}{c}\text { Double } \\
\text { non-linear } \\
\text { algorithm }\end{array}$ & $\begin{array}{c}\text { Nearest } \\
\text { neighbor } \\
\text { algorithm }\end{array}$ \\
\hline \multirow{2}{*}{ Lena } & 2 & 43.03 & 63.91 & 83.61 & 97.07 \\
& 4 & 141.43 & 178.32 & 207.36 & 256.86 \\
& 8 & 386.57 & 387.16 & 432.36 & 501.53 \\
Couple & 16 & 639.58 & 697.07 & 769.01 & 814.71 \\
& 2 & 8.68 & 59.06 & 71.03 & 84.20 \\
& 4 & 139.97 & 140.60 & 158.07 & 181.98 \\
\hline \hline
\end{tabular}


It can be seen from Table 2 that after the 2, 4, 8 and 16 times of reduction, the interpolation algorithm enlarges to the original size. The mean square error of the interpolation algorithm proposed in this paper is the smallest, which also shows that the interpolation algorithm in this paper is the most stable and has the most obvious effect. As can be seen from Table 3 , the interpolation algorithm proposed in this paper needs less computation time than the other three interpolation algorithms, and it can be known from the experimental results that the interpolation algorithm in this paper not only has the best interpolation effect but also can provide real-time processing guarantee.. As can be seen from Table 4, the interpolation method proposed in this paper obtains the best SSIM index, and objectively it interpolates the highest image visual quality, and the interpolation effect is also the best.

Table 3. Different magnification algorithm different calculation time (unit: second) statistics

\begin{tabular}{cccccc}
\hline \hline $\begin{array}{c}\text { Experimental } \\
\text { images }\end{array}$ & Gain & $\begin{array}{c}\text { This } \\
\text { paper's } \\
\text { method }\end{array}$ & $\begin{array}{c}\text { Double } \\
\text { three times } \\
\text { algorithm }\end{array}$ & $\begin{array}{c}\text { Double } \\
\text { non-linear } \\
\text { algorithm }\end{array}$ & $\begin{array}{c}\text { Nearest } \\
\text { neighbor } \\
\text { algorithm }\end{array}$ \\
\hline \multirow{3}{*}{ Lena } & 2 & 0.25 & 0.19 & 0.18 & 0.17 \\
& 4 & 0.18 & 0.19 & 0.18 & 0.17 \\
& 8 & 0.26 & 0.21 & 0.19 & 0.19 \\
Couple & 16 & 0.19 & 0.20 & 0.17 & 0.16 \\
& 2 & 0.29 & 0.21 & 0.21 & 0.21 \\
& 4 & 0.27 & 0.22 & 0.20 & 0.20 \\
\hline \hline
\end{tabular}

Table 4. SSIM statistics for different algorithms for different magnifications

\begin{tabular}{cccccc}
\hline \hline $\begin{array}{c}\text { Experimental } \\
\text { images }\end{array}$ & Gain & $\begin{array}{c}\text { This } \\
\text { paper's } \\
\text { method }\end{array}$ & $\begin{array}{c}\text { Double } \\
\text { three times } \\
\text { algorithm }\end{array}$ & $\begin{array}{c}\text { Double } \\
\text { non-linear } \\
\text { algorithm }\end{array}$ & $\begin{array}{c}\text { Nearest } \\
\text { neighbor } \\
\text { algorithm }\end{array}$ \\
\hline \multirow{2}{*}{ Lena } & 2 & 0.9675 & 0.9378 & 0.9003 & 0.8912 \\
& 4 & 0.9421 & 0.9026 & 0.8767 & 0.8591 \\
& 8 & 0.9076 & 0.8985 & 0.8446 & 0.7901 \\
Couple & 16 & 0.8319 & 0.8235 & 0.7741 & 0.7367 \\
& 2 & 0.9587 & 0.9082 & 0.9001 & 0.8991 \\
& 4 & 0.9336 & 0.8929 & 0.8863 & 0.8877 \\
\hline \hline
\end{tabular}

\subsection{Experiment 2}

To better serve the mine measured image processing, this example mine support image using the method proposed in this paper and the wavelet method to enhance the processing, and is shown in Fig. 16. This method of image interpolation enhances the image. The effect is shown in Fig. 17, and the wavelet method for image enhancement effect is shown in Fig. 18.

It can be seen from Fig. $\mathbf{1 6}$ to Fig. 18 that the image obtained by the method of the present invention can clearly identify the texture information of the image. The image obtained by the wavelet method cannot well recognize the texture information contained in the image.

To analyze the above method in the aspect of preserving the image feature information, the indicators such as the PSNR, the MSE and the SSIM of the images obtained by the two methods are analyzed in detail as shown in Table 5 to Table 7 below. 

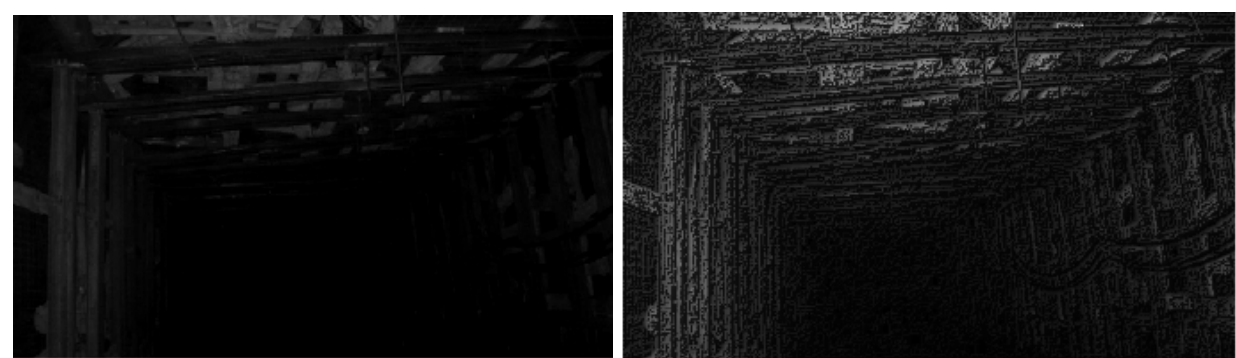

Fig. 16. Measured roadway support grayscale Fig. 17. This method interpolates the enhanced renderings

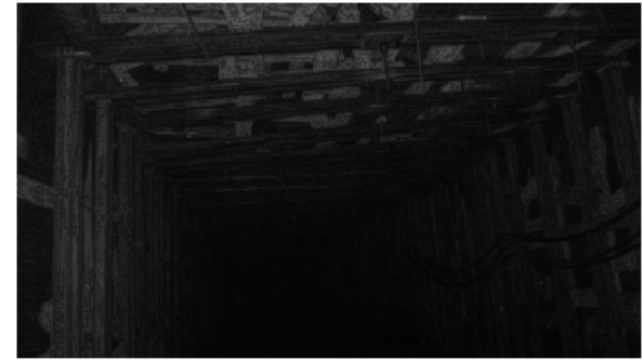

Fig. 18. Wavelet method to enhance the effect map

Table 5. PSNR (unit: dB) statistics table of two methods after the measured image processing

\begin{tabular}{ccc}
\hline \hline Image type & $\begin{array}{c}\text { This } \\
\text { paper's } \\
\text { method }\end{array}$ & $\begin{array}{c}\text { Wavelet } \\
\text { method }\end{array}$ \\
\hline PSNR & 43.25 & 39.37 \\
\hline \hline
\end{tabular}

Table 6. two methods for the measured image processing MSE statistical tables

\begin{tabular}{ccc}
\hline \hline Image type & $\begin{array}{c}\text { This } \\
\text { paper's } \\
\text { method }\end{array}$ & $\begin{array}{c}\text { Wavelet } \\
\text { method }\end{array}$ \\
\hline MSE & 69.31 & 78.92 \\
\hline \hline
\end{tabular}

Table 7. Two methods for the measured image processing ssim statistical tables

\begin{tabular}{ccc}
\hline Image type & $\begin{array}{c}\text { This } \\
\text { paper's } \\
\text { method }\end{array}$ & $\begin{array}{l}\text { Wavelet } \\
\text { method }\end{array}$ \\
\hline SSIM & 0.8961 & 0.8503 \\
\hline \hline
\end{tabular}

It can be seen from Table 5 to Table 7 that the peak SNR of the image obtained by the proposed method is the highest, which also objectively shows the superiority of the proposed method in the processing of the down hole measured image features. The mean square error of the image obtained by this method is small, which also reflects that the measured image is more stable and the effect is the most significant. It can also provide more favorable image feature information support for the surrounding rock deformation of mine roadway in the later period. The SSIM of interpolation results obtained by this method is the best, which shows that the interpolation image obtained has the best visual effect.

\subsection{Experiment 3}

To better reflect the advantages of the interpolation method proposed in this paper, this experiment selects the cameraman image from the standard test image library to conduct 
experiments, and the experimental images are shown in Fig. 19. In the experimental process, first, the image is reduced by 4 times. Then the interpolation algorithm [35, 36] are used to respectively conduct the size reduction, and the obtained reduction effect is shown in Fig. 20. Moreover, the PSNR, MSE and SSIM of the interpolated image are calculated, as shown in Table 8.

Table 8. PSNR, MSE and SSIM statistics of different magnification and different algorithms

\begin{tabular}{cccc}
\hline $\begin{array}{c}\text { Indicator } \\
\text { type }\end{array}$ & $\begin{array}{c}\text { This paper } \\
\text { method }\end{array}$ & $\begin{array}{c}\text { Reference } \\
{[35]}\end{array}$ & Reference [36] \\
\hline PSNR & 29.69 & 27.37 & 26.39 \\
MSE & 128.43 & 120.03 & 121.07 \\
SSIM & 0.9482 & 0.9018 & 0.9187 \\
\hline
\end{tabular}

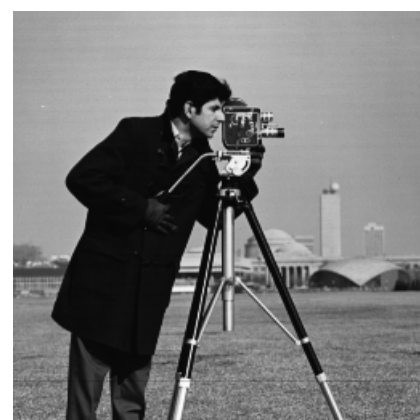

Fig. 19. Cameraman

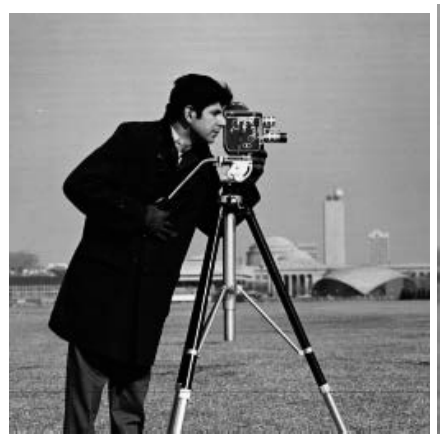

(a) Fractal Interpolation

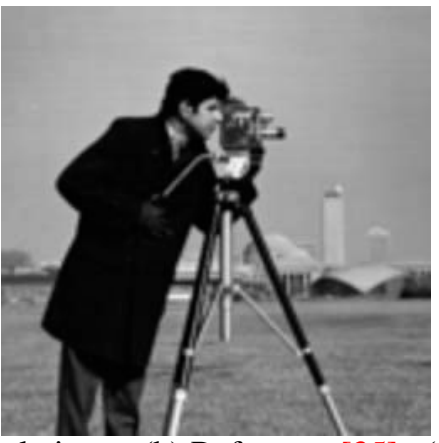

(b) Reference [35]

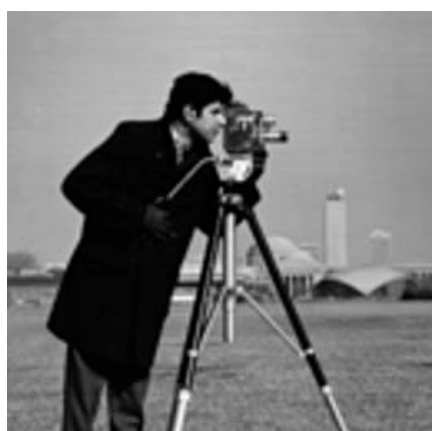

(c) Reference [36]

Fig. 20. Different interpolation methods enlarge the image to the original size effect diagram after reducing the image by $4^{\text {th }}$

For the cameraman images, the interpolation method in this paper and References [35-36] are respectively used to interpolate and enlarge to the original size after reducing it by 4 . As you can see from Fig. 20, the method proposed in this paper is superior to the interpolation method in References [35-36] with respect to subjective vision. As seen from Table 6, the MSE of interpolation results obtained by this method is the smallest, which reflects that the interpolation algorithm in this paper is the most stable. The PSNR of the interpolation results obtained by this method is the highest, which indicates that the interpolation image obtained is of the best quality. The SSIM of the interpolation results obtained by this method is the best, which shows that the interpolation image obtained has the best visual effect.

The experimental results from Experiment 1 to 3 show that the interpolation method proposed in this paper is optimal after 2nd, 4th, 8th, and 16th scale reduction and then 
amplified to the original size. This finding is primarily observed because the fractal interpolation method proposed in this paper first analyzes the amount of image feature information, and then it performs interpolation according to the information of the image feature information. This result is equivalent to understanding the size distribution of the feature information contained in the image pixels, and interpolation is subsequently performed. This approach can improve the utilization of feature information in the interpolation process, and fully implement data-driven interpolation according to the self-characteristic information of the image itself. This method is also fundamentally different from other interpolation methods, and it can ensure the interpolation effect of the image in principle.

\section{Conclusion}

This work proposes an adaptive fractal interpolation algorithm, in which all parameters are determined by the particle swarm optimization. The algorithm aims to solve the problem of inaccurate and low real-time interpolation in bi-dimensional empirical mode decomposition, for the problem may cause a reliable bi-dimensional intrinsic mode function. The proposed algorithm not only improves the interpolation accuracy, but also inceases the interpolation efficiency. Some case analyses illlustrate its advantage over the other interpolation algorithms.

Experiments show that the Lena and Couple images are magnified to the original size after being reduced in size by 2, 4, 8 and 16 times. This paper proposes that after 8 and 16 times of the algorithm, the interpolated renderings are obviously superior to the other three algorithms Especially in the case of 16 times, the renderings obtained by the interpolation algorithm in this paper are still relatively clear. The renderings obtained by the other three algorithms are already quite vague, especially the result graph obtained by the nearest neighbor interpolation method can not be distinguished The image of the character information, we can see the advantage of this algorithm is more obvious. In the case of 2,4 times, the algorithm in this paper also has advantages, but the subjective visual perception is not obvious. Follow-up will be analyzed in detail through objective indicators such as peak signal-noise ratio and mean square error. The proposed algorithm not only has the highest peak signal to noise ratio, but also has the smallest mean square error, which verifies the advantages of the interpolation algorithm in this paper. In addition, it can be known from the experimental results that the interpolation method proposed in this paper is better than the bilinear, adjacent and other interpolation methods, and is also better than the interpolation methods proposed in recent years, such as those in References [35-36]. This is mainly because the interpolation method proposed in this paper makes full use of the characteristic information of the image to be interpolated, and then performs the interpolation. It is an interpolation method based on the information of the image itself. This is the essential difference between and the interpolation method proposed in this paper and other interpolation methods, and it is also the key cause of its best interpolation effect.

It can be seen from Fig. 16, 17, 18 and 20 that for the measured image interpolation, the image obtained by the method of the present invention can clearly identify the texture information. The image obtained by the wavelet method can not well recognize the texture information contained in the image. Furthermore, the PNSR, the MSE, and the SSIM of the image obtained by the method proposed in this paper are also small. This result also reflects that the measured image is more stable and the effect is the most obvious. It can provide more favorable image feature information support for the surrounding rock deformation of the mine roadway in the later period. 


\section{Acknowledgment}

This work is supported by National Science Foundation Project of P. R. China (No. 61701188). This work was supported by the Natural Science Foundation of Jiangsu Province (Grants No BK20141253), and Qing Lan Project of Jiangsu Province.

\section{References}

[1] Nunes J C, Bouaouue Y, Delechelle E, "Image analysis by bi-dimensional empirical mode decomposition,” Image Vision Computing, vol. 21, no. 12, pp. 1019-1026, Nov. 2003. Article (CrossRef Link)

[2] Nunes J C, Guyot S, Delechelle. E, "Texture analysis based on local analysis of the bi-dimensional empirical mode decomposition," Machine Vision and Applications, vol. 16, no. 3, pp. 177-188, May. 2005. Article (CrossRef Link)

[3] Zhou Y, Li H, "Adaptive noise reduction method for DSPI fringes based on bi-dimensional ensemble empirical mode decomposition,” Optics express, vol. 19, no. 19, pp. 18207-18215, Sep. 2011. Article (CrossRef Link)

[4] Rubin S G Khosla P K, "Polynomial interpolation methods for viscous flow calculations,” Journal of Computational Physics, vol. 24, no. 3, pp. 217-244, July. 1977. Article (CrossRef Link)

[5] Akima $\mathrm{H}$, Gebhardt A, Petzoldt T, “akima: Interpolation of irregularly spaced data," $R$ package version 0.5-11, Nov. 16, 2013. Article (CrossRef Link)

[6] De Boor C, Höllig K, Sabin M, "High accuracy geometric Hermite interpolation," Computer Aided Geometric Design, vol. 4, no. 4, pp. 269-278, Dec. 1987. Article (CrossRef Link)

[7] He Z, Wang Q, Shen Y, "Multivariate gray model-based BEMD for hyperspectral image classification," IEEE Transactions on Instrumentation and Measurement, vol. 62, no. 5, pp. 889-904, May. 2013. Article (CrossRef Link)

[8] Zhao J, Zhao P, Chen Y, "Using an improved BEMD method to analyse the characteristic scale of aeromagnetic data in the Gejiu region of Yunnan, China," Computers \& Geosciences, vol. 88, no. 2016, pp. 132-141, Mar. 2016. Article (CrossRef Link)

[9] Li T, Wang Y, "Biological image fusion using a NSCT based variable-weight method," Information Fusion, vol. 12, no. 2, pp. 85-92, April. 2011. Article (CrossRef Link)

[10] Yin S, Cao L, Ling Y, "One color contrast enhanced infrared and visible image fusion method," Infrared Physics\&Teehnology, vol. 53, no. 2, pp. 146-150, Mar. 2010. Article (CrossRef Link)

[11] Lin D C, Guo Z L, An F P, "Elimination of end effects in empirical mode decomposition by mirror image coupled with support vector regression,” Mechanical Systems and Signal Processing, vol. 31, no. 1, pp. 13-28, Aug. 2012. Article (CrossRef Link)

[12] Linderhed A, "Variable sampling of the empirical mode decomposition of two-dimensional signals," International journal of wavelets, multiresolution and information processing, vol. 3, no. 3, pp. 435-452, Sep. 2005. Article (CrossRef Link)

[13] Wu Z, Huang N E, Chen X, "The multi-dimensional ensemble empirical mode decomposition method," Advances in Adaptive Data Analysis, vol.1, no. 3, pp. 339-372, July. 2009. Article (CrossRef Link)

[14] Bernini M B, Federico A, Kaufmann G H, "Noise reduction in digital speckle pattern interferometry using bi-dimensional empirical mode decomposition," Applied optics, vol. 47, no. 14, pp. 2592-2598, May. 2008. Article (CrossRef Link)

[15] Wielgus M, Patorski K, "Evaluation of amplitude encoded fringe patterns using the bi-dimensional empirical mode decomposition and the 2D Hilbert transform generalizations," Applied optics, vol. 50, no. 28, pp. 5513-5523, Sep. 2011. Article (CrossRef Link)

[16] Bhuiyan S M A, Attoh-Okine N O, Barner K E, "Bi-dimensional empirical mode decomposition using various interpolation techniques," Advances in Adaptive Data Analysis, vol. 1, no. 2, pp. 309-338, April. 2009. Article (CrossRef Link) 
[17] Bernini M B, Federico A, Kaufmann G H, "Normalization of fringe patterns using the bi-dimensional empirical mode decomposition and the Hilbert transform,” Applied optics, vol. 48, no. 36, pp. 6862-6869, Dec. 2009. Article (CrossRef Link)

[18] Liu Z, Wang H, Peng S, “Texture segmentation using directional empirical mode decomposition,” in. Proc. of ICIP'04, USA, pp. 279-282, 2004. Article (CrossRef Link)

[19] Lei D, Xiaolin H, Feng L, "Support vector machines-based method for restraining end effects of B-spline empirical mode decomposition,” Journal of Vibration, Measurement \& Diagnosis, vol. 31, no. 3, pp. 344-347, 3: 1-19, 2011. Article (CrossRef Link)

[20] Al-Baddai S, Al-Subari K, Tomé A M, “A Green’s function-based bi-dimensional empirical mode decomposition,” Information Sciences, vol. 348, no. 2016, pp. 305-321, June. 2016. Article (CrossRef Link).

[21] Xu G, Cheng Q, Zuo R, “Application of improved bi-dimensional empirical mode decomposition (BEMD) based on Perona-Malik to identify copper anomaly association in the southwestern Fujian,” Journal of Geochemical Exploration, vol. 164, no. 2016, pp. 65-74, May. 2016. Article (CrossRef Link).

[22] Falconer K, "Fractal geometry: mathematical foundations and applications," New Jersey, USA: John Wiley \& Sons, pp. 109-139, 2004.

[23] Falconer K, "Fractals: Theory and Applications in Engineering: Theory and Applications in Engineering,” Berlin, Germany: Springer Science \& Business Media, pp. 201-289, 2012.

[24] Torres I C, Rubio J M A, Ipsen R, "Using fractal image analysis to characterize microstructure of low-fat stirred yoghurt manufactured with microparticulated whey protein," Journal of Food Engineering, vol. 109, no. 4, pp. 721-729,April. 2012. Article (CrossRef Link)

[25] Clerc M, “Particle swarm optimization,” New Jersey, USA: John Wiley \& Sons, pp. 321-389, 2010.

[26] Ke Y, Sukthankar R, "PCA-SIFT: A more distinctive representation for local image descriptors," in Proc. of Proceedings of the 2004 IEEE Computer Society Conference on. IEEE, USA, pp. 506-513, 2004.Article (CrossRef Link)

[27] Grefenstette J J, “Genetic Algorithms and Their Applications,” in Proc. of Proceedings of the Second International Conference on Genetic Algorithms, London, England: Psychology Press, 2013, pp. 309-337, 2013. Article (CrossRef Link)

[28] Goldberg D E, “The design of innovation: Lessons from and for competent genetic algorithms,” Germany: Springer Science \& Business Media, pp. 138-165, 2013. Article (CrossRef Link)

[29] Steeb W H, "The nonlinear workbook: chaos, fractals, cellular automata, genetic algorithms, gene expression programming, support vector machine, wavelets, hidden Markov models, fuzzy logic with C++, Java and SymbolicC++ programs," Singapore: World Scientific Publishing Co Inc, pp. 307-334, 2014. Article (CrossRef Link)

[30] Sastry K, Goldberg D E, Kendall G, “Genetic algorithms,” Germany: Springer US, 93-117, 2014. Article (CrossRef Link)

[31] Scrucca L, “GA: a package for genetic algorithms in R,” Journal of Statistical Software, vol. 53, no. 4, pp. 1-37, 2013. Article (CrossRef Link)

[32] Panichella A, Dit B, Oliveto R, "How to effectively use topic models for software engineering tasks? an approach based on genetic algorithms," in Proc. of Proceedings of the 2013 International Conference on Software Engineering, USA, pp. 522-531, 2013. Article (CrossRef Link)

[33] Grefenstette J J, “Genetic Algorithms and Their Applications," in Proc. of Proceedings of the Second International Conference on Genetic Algorithms, London, England: Psychology Press, pp. 109-138, 2013. Article (CrossRef Link)

[34] Wang Z, Bovik A C, "Mean squared error: Love it or leave it? A new look at signal fidelity measures,” IEEE signal processing magazine, vol. 26, no. 1, pp. 98-117, 2009. Article (CrossRef Link)

[35] Sadeghi B, Yu R, Wang R, “Shifting Interpolation Kernel toward Orthogonal Projection,” IEEE Transactions on Signal Processing, vol. 66, no. 1, pp. 101-112, 2018. Article (CrossRef Link) 
[36] Bornert M, Doumalin P, Dupré J C, "Shortcut in DIC error assessment induced by image interpolation used for subpixel shifting," Optics and Lasers in Engineering, no. 91, pp. 124-133, 2017. Article (CrossRef Link)

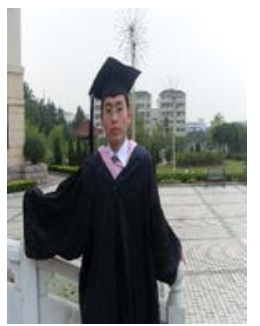

Fengping An received the B.S. degree in School of Economic and Management from Hefei University, Hefei, China, in 2008 and the M.S. degree in School of Economics and Management from Hebei University of Engineering, Handan, China, in 2011 and the Ph.D. degree in School of Computer and Communication Engineering from Beijing University of Science and Technology, China, in 2017. He has worked as a postdoctoral researcher in Beijing Institute of Technology. He has worked in Huaiyin Normal of University. He has research areas of Image Processing, Artificial Intelligence and Pattern Recognition.

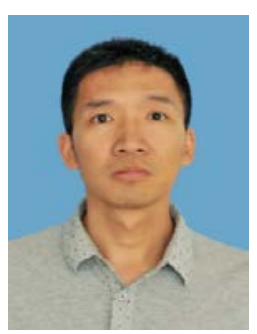

Xin He was born in Henan, China, in 1974. He received the B.S. and M.S. degrees in computer science from the School of Computer and Information Engineering, Henan University, Kaifeng, China, and the Ph.D. degree in computer science from Xi'an Jiaotong University, Xi'an, China, 2011. He has been teaching in Henan University. His research interests include network computing, crowd sensing and image processing. 